\title{
A porcine model for pathomorphological age assessment of surgically excised skin wounds
}

\author{
Kristiane Barington ${ }^{*} \mathbb{0}$, Kristine Dich-Jørgensen and Henrik Elvang Jensen
}

\begin{abstract}
A porcine model of second intention wound healing was established and gross and histological changes needed for accurate assessing the age of wounds were determined. Twenty-five pigs were anesthetized before incision of four wounds on each. The wounds were left to heal from $1 \mathrm{~h}$ to 35 days when the pigs were euthanized. In 14 pigs, biopsies were sampled from two wounds between days 2 and 18. By histological evaluation the following characteristics were found useful for determining the age of wounds: neutrophil:macrophage ratio, angiogenesis, hyperplasia of fibroblasts, presence of hemosiderophages and granulation tissue. The latter was present from day 4 ( $n=8$ wounds, $100 \%)$, but by gross evaluation it was not recognized until day 5 ( $n=4$ wounds, $100 \%)$. From day 4 to 10 , the thickness of granulation tissue increased by $1.2 \pm 2.4 \mathrm{~mm} /$ day. The thickness of collagen fibers within granulation tissue increased throughout the study period, and complete epithelization was reached from day 18.
\end{abstract}

Keywords: Age of wounds, Experimental animal model, Pig, Skin, Ulceration, Veterinary forensic pathology

\section{Findings}

Age assessment of skin wounds in pigs is often requested in veterinary forensic pathology $[1,2]$. Wounds in pigs are primarily caused by external trauma, and the age of wounds submitted for forensic investigation ranges from hours and up to several months [3]. Methods to determine the age of wounds have been investigated in porcine models [4-6]. However, in previous studies and unlike wounds in veterinary forensic cases, these wounds were treated with a epinephrine solution to obtain hemostasis or bandaged during healing [4-6]. The aim of the present study was to identify hallmarks for forensic age assessment of porcine wounds by assessing characteristics in experimental wounds during healing by second intention.

Twenty-five specific pathogen free (SPF) female Yorkshire-Landrace crossbred pigs with body weights of 23-34 $\mathrm{kg}$ were acclimatized for 1 week, housed

\footnotetext{
*Correspondence: krisb@sund.ku.dk

Department of Veterinary and Animal Sciences, Faculty of Health

and Medical Sciences, University of Copenhagen, Ridebanevej 3,

1870 Frederiksberg C, Denmark
}

individually with bedding of straw and sawdust (Spanvall, Denmark) and numbered 1-25 in the order they arrived to the facility (Table 1).

Each pig was anesthetized (Additional file 1), treated with continuous intravascular infusion of fentanyl (Fentanyl $50 \mu \mathrm{g} / \mathrm{mL}$, Fentanyl-Hameln; Hameln Pharmaceuticals gmbh, Hameln, Germany), placed in sternal recumbency and prepared for sterile surgery. Four surgical areas of $2 \times 2 \mathrm{~cm}$ (locations 1-4) were drawn on the back in the paravertebral area (Fig. 1). By incision, full thickness wounds (from the epidermis down to and including the subcutis) were established by removal of $4 \mathrm{~cm}^{2}$ skin and left to heal by second intention. Pigs $1-5$ were kept anesthetized for 1-6 $\mathrm{h}$ and then euthanized, while pigs 6-25 were transported to their pens to wake up (Table 1). At $8 \mathrm{~h}$ intervals, pigs were given four injections of buprenorphine (Temgesic, $0.3 \mathrm{mg} / \mathrm{mL}$, ScheringPlough, NJ, USA). None of the pigs showed sign of pain as no increase in respiratory rate, depression, reduced feed intake or mobility were observed. At different time points, pigs 12-25 were anesthetized (Additional file 2) before two full thickness biopsies from each of the wound margins of locations 2 and 3 were sampled by incision 


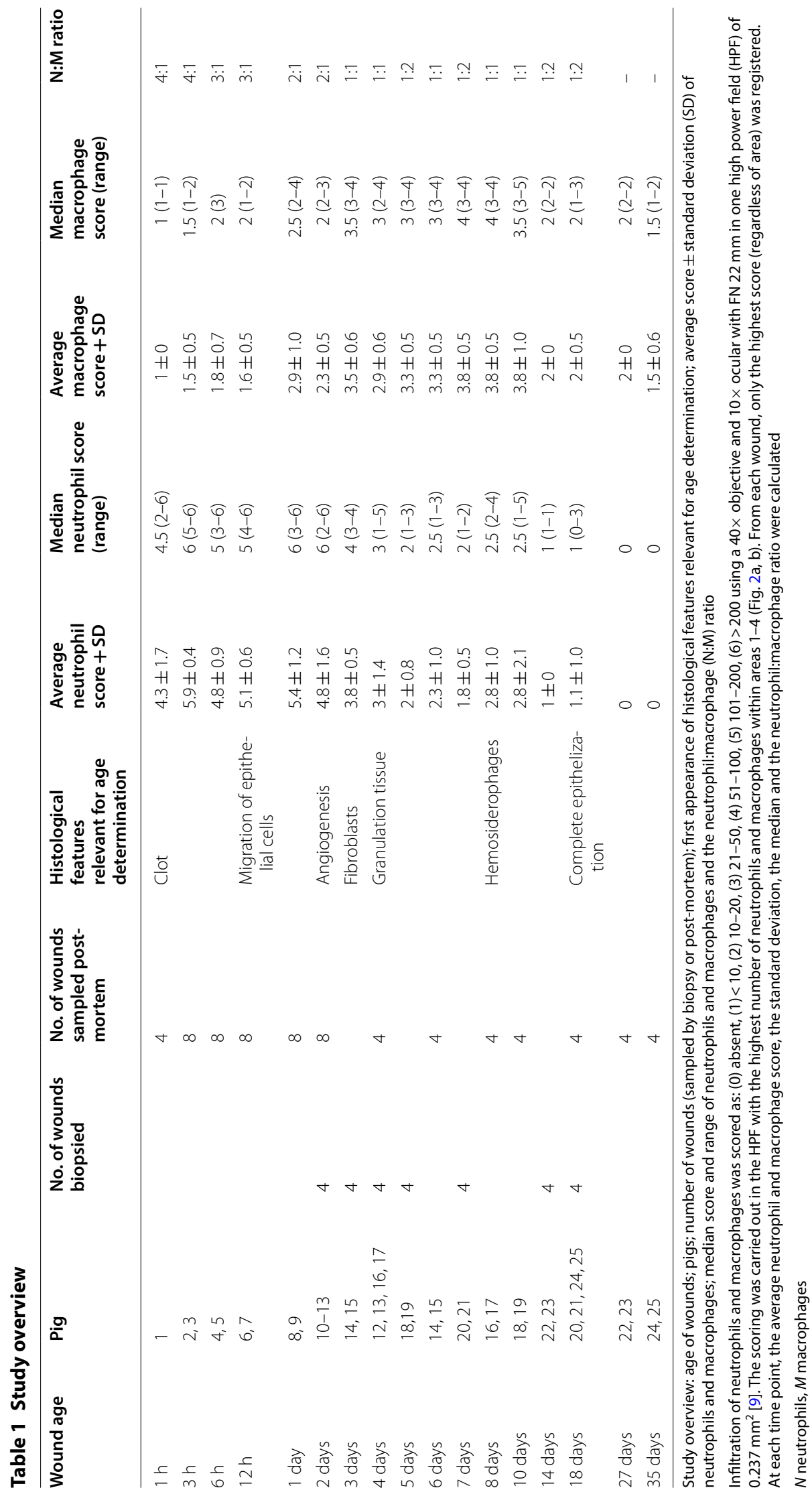




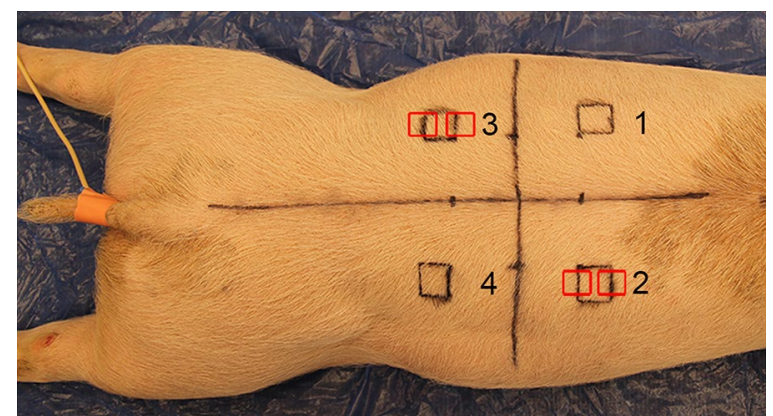

Fig. 1 Location of wounds 1-4, experimental pig 21. Each wound was located $4 \mathrm{~cm}$ lateral to the spine and $4 \mathrm{~cm}$ cranial or caudal to the last rib, respectively. Biopsies were taken from two wound edges at locations 2 and 3 (red boxes) while the pigs were anesthetized. Wounds at location 1 and 4 were sampled post mortem

(Fig. 1 and Table 1). After $4 \mathrm{~h}$, these pigs received an intramuscular injection of $0.1 \mathrm{mg} / \mathrm{kg}$ buprenorphine. The pigs were euthanized from $1 \mathrm{~h}$ to 35 days after the creation of the wounds with an overdose of intravenous pentobarbital (Glostrup Apotek, Glostrup, Denmark) (Table 1).

Following euthanasia, cross sections of the wounds that had not previously been biopsied were sampled for histology. In total, 100 wounds were evaluated, i.e. 28 wounds were biopsied and 72 wounds were sampled after euthanasia (Table 1). Granulation tissue was defined as a red and hemorrhagic connective tissue filling the wound bed and recorded as present or absent by gross inspection [7]. In wounds that were biopsied, evaluations were only recorded until the day of biopsy sampling.

Histological preparation of the tissue was done as previously described [8]. Sections stained with hematoxylin and eosin were evaluated in four areas $(3 \times 3 \mathrm{~mm})$ depending on the age of the wounds and therefore not blinded (Fig. 2a, b). All registrations were carried out by a single veterinary pathologist. Infiltration of neutrophils and macrophages was scored as: (0) absent, (1)<10; (2) 10-20; (3) 21-50; (4) 51-100; (5) 101-200; (6) $>200$ using a $40 \times$ objective and $10 \times$ ocular with FN $22 \mathrm{~mm}$ in one high power field (HPF) of $0.237 \mathrm{~mm}^{2}$ [9]. The scoring was carried out in the HPF with the highest number of neutrophils and macrophages within areas 1-4 (Fig. 2a, b). From each wound, only the highest score (regardless of area) was registered, i.e. one score per wound. Similarly, when two biopsies were sampled from one wound both biopsies were evaluated, but only the highest score was registered. At each time point, the average neutrophil and macrophage score, the standard deviation (SD), the median and the neutrophil:macrophage ratio were calculated. Hemorrhage, hyperplasia of fibroblasts and endothelial cells and angiogenesis were registered as present or absent in areas 1-4 (Figs. 2a, b). Granulation tissue was defined as fibroblasts and collagen arranged

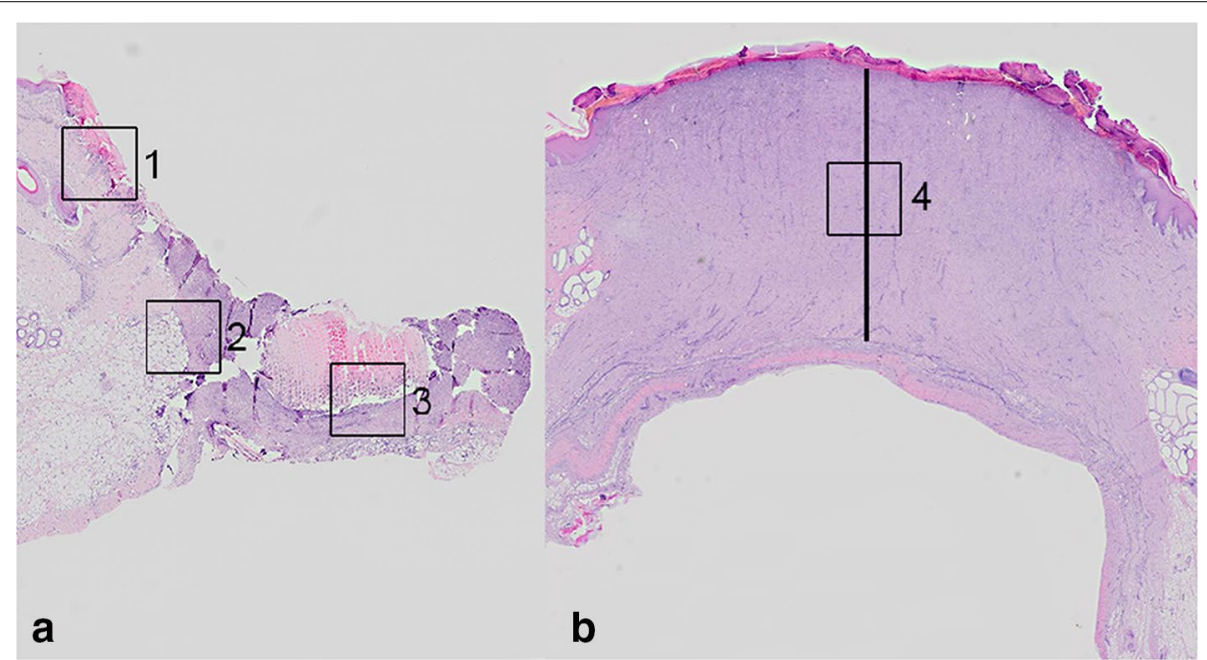

Fig. 2 Areas subjected to histological evaluation of biopsies (a) or cross sections of wounds (b), hematoxylin and eosin stain. a Histological evaluation of wounds aged $1 \mathrm{~h}$ to 10 days was carried out in areas 1-3 (3 mm $\times 3 \mathrm{~mm})$. Area 1: at the wound edge, dermis. Area 2: at the wound edge, subcutis. Area 3: central in the wound bed. b Histological evaluation of wounds aged 14 to 35 days was carried out in area 4 ( $3 \mathrm{~mm} \times 3 \mathrm{~mm}$ ), i.e. central in the granulation tissue. The thickness of the granulation tissue was measured at the center of the wound (straight line) 


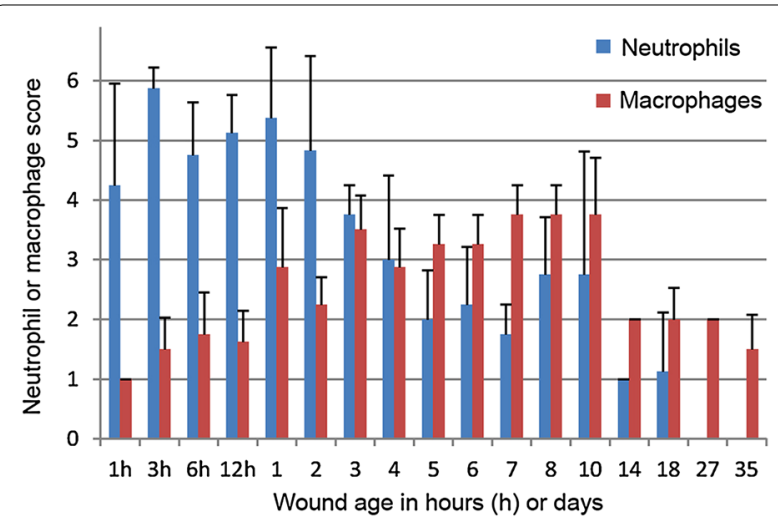

Fig. 3 The average score \pm standard deviation of neutrophils and macrophages in wounds being from $1 \mathrm{~h}$ to 35 days

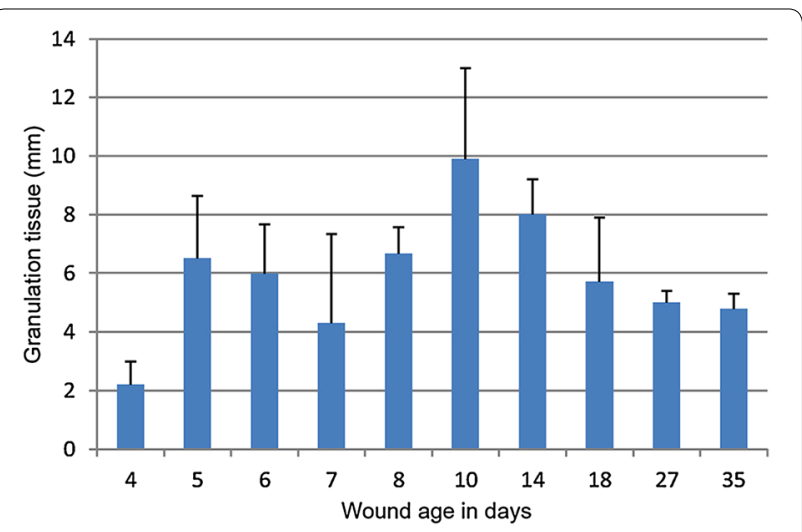

Fig. 4 The average thickness \pm standard deviation of granulation tissue in wounds being from 4 to 35 days perpendicular to the new proliferating vessels (angiogenesis) [7]. When present, the thickness of granulation tissue was measured at the center of the wound from the top of the wound bed (not including epidermis) to the level of normal tissue components using the software Delta Pix 2.3.5 (Smorum, Denmark) (Fig. 2b). Differences in the thickness of granulation tissue, due to the location of the wound (location 1-4), were evaluated by comparing locations 1 and 4 and locations 2 and 3 using the paired $t$ test $(\mathrm{P}<0.05)$. Moreover, at each time point the mean thickness of granulation tissue and SD were calculated (Additional file 3).

Epithelization was characterized as: (0) absent, (1) hyperplasia of epithelial basal cells, (2) migration of epithelial cells, and (3) full epithelization.

Randomly selected sections from all time points were stained with Perl's Prussian blue, Masson's trichrome, and Picrosirius red in order to confirm the presence of hemosiderophages and visualization of collagen in the wound bed $[10,11]$. The change in polarization color from green to yellow to red in the aging wounds stained with Picrosirius red is due to increasing thickness of collagen [11]. Immunohistochemistry based on polyclonal rabbit antihuman von Willebrand Factor antibody (A0082, Agilent, USA) was used to confirm the presence of vessels [12].

The first appearance of the most important histological manifestations for determining the age of wounds is presented in Table 1. Hemostasis was present within $1 \mathrm{~h}$ where a clot had formed in the wound bed. Infiltration of neutrophils around vessels and in the dermal and subcutaneous tissue was seen from $1 \mathrm{~h}$ to day 18 (90 of 92 wounds, 98\%). In comparison, a intravital reaction (i.e. active infiltration of leukocytes) is not found in porcine wounds inflicted postmortem or seconds before death $[8$, 13]. The number of neutrophils peaked after $3 \mathrm{~h}$ to 2 days with a score greater than 4.5 (Fig. 3) (Additional file 4).

Infiltration of macrophages in the dermis and subcutis was seen in the wounds ( $\mathrm{n}=100$ wounds, $100 \%)$ throughout the experimental period but peaked from day 7 to 10 with a score greater than 3.5 (Fig. 3) (Additional file 4). In accordance with this, macrophages peaked at day 7 in wounds in a previous porcine excisional wound model [14]. From 8 to 35 days, hemosiderophages were present. In comparison, hemosiderophages, which to the authors knowledge have not been noticed in porcine wounds hitherto, have been reported in human wounds already at day 3 and appeared regularly from day 8 [15].

Based on the neutrophil:macrophage ratio, wounds could roughly be assessed as being from 1 to $3 \mathrm{~h}, 6$ to $12 \mathrm{~h}, 1$ to 2 days or more than 3 days (Table 1 ). Contamination of the wounds with bedding material was inevitable and may have affected the inflammatory reaction [16]. However, in forensic cases, wounds are also subjected to infection, foreign bodies and continuous trauma, which can affect the neutrophil:macrophage ratio [16]. Hyperplasia of endothelial cells, angiogenesis and hyperplasia 


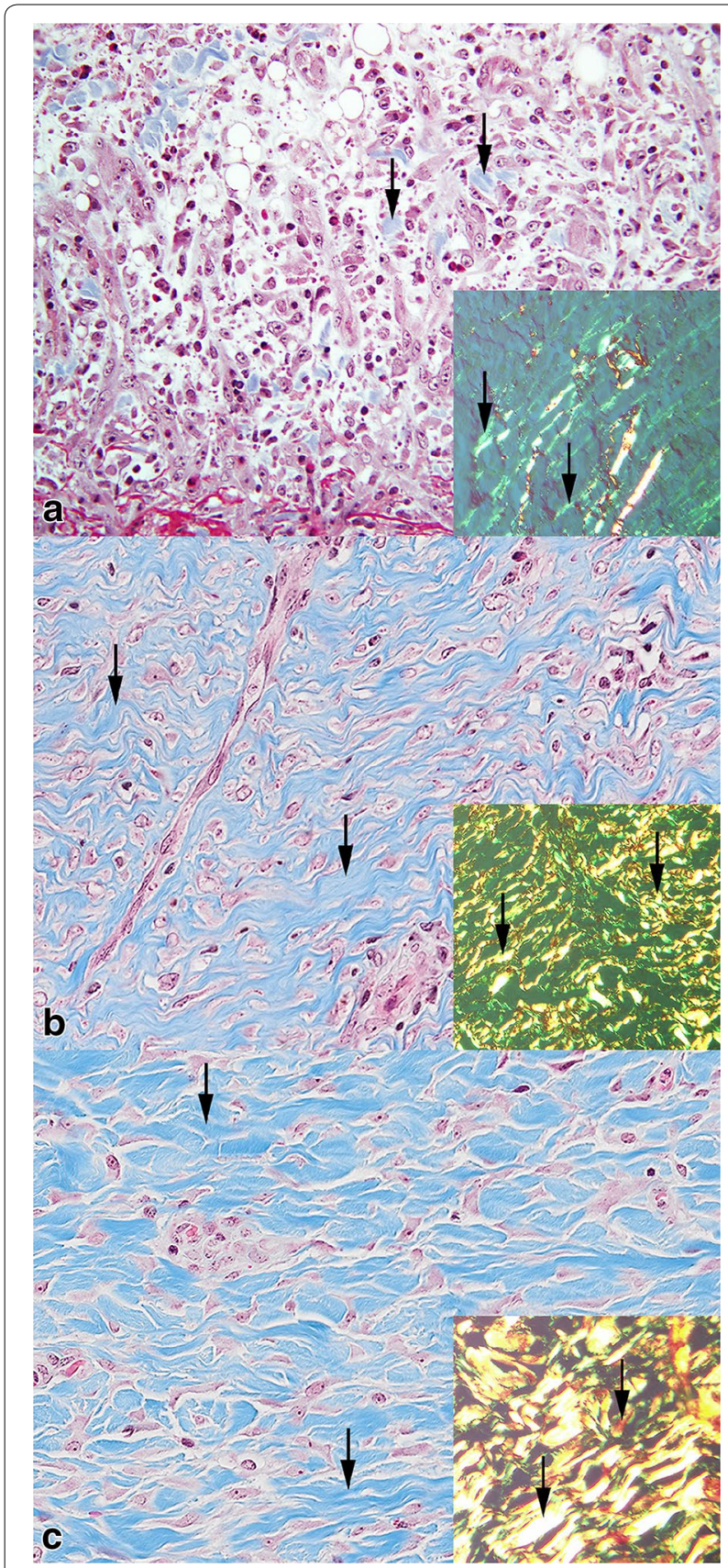

Fig. 5 Porcine wound beds being 4 days (a), 10 days (b) and 27 days (c) old. a A scarce amount of blue stained collagen is present (arrows), Masson's trichrome stain. Inset: Green collagen fibers are visible under polarized light (arrows), Picrosirius red stain. b Massive amounts of blue stained collagen are present (arrows), Masson's trichrome stain. Inset: Green, yellow and few red collagen fibers are visible in polarized light (arrows), Picrosirius red stain. c Massive amounts of blue stained collagen are present (arrows), Masson's trichrome stain. Inset:Yellow, red and a few green collagen fibers are visible in polarized light (arrows), Picrosirius red stain of fibroblast were observed in all wounds from $12 \mathrm{~h}, 2$ and 3 days, respectively. Our observations are in accordance with previous records on experimental wounds in pigs, in which hyperplasia of fibroblasts was seen within 3 days [17]. Moreover, in forensic porcine wounds, we have previously predicted angiogenesis and hyperplasia of fibroblasts to begin at $16 \mathrm{~h}$ to 3 days [3].

Histologically, granulation tissue was present at day 4 ( $\mathrm{n}=8$ wounds, $100 \%$ ), but at gross evaluation it was not recognized until day $5(\mathrm{n}=4$ wounds, $100 \%)$. Granulation tissue thickness on days 4 to 7 varied from approximately $2-6 \mathrm{~mm}$ and increased to nearly $10 \mathrm{~mm}$ on day 10 (growth rate: $1.2 \pm 2.4 \mathrm{~mm}$ per day). However, from day 10 to 35 the thickness decreased by $0.3 \pm 0.3 \mathrm{~mm}$ per day (Fig. 4) (Additional file 3). In forensic cases of porcine wounds, granulation tissue with thicknesses of 5 , 15 and $27 \mathrm{~mm}$ have been used to estimate wound age as being 4-7 days, $8-28$ days and $>28$ days, respectively [3]. The decrease in granulation tissue thickness seen in the experimental wounds but apparently not in forensic cases is likely due to the initial depth of the lesion, accompanying infection, sequestration of necrotic tissue and sustained injury [16]. Therefore, the growth rate of granulation tissue up to 10 days should probably be seen as the maximum by which it can be formed. The wide SD of the average growth rate is likely due to inter pig variation as no differences in granulation tissue thickness between locations 1 and $4(\mathrm{P}=0.26)$ and locations 2 and $3(\mathrm{P}=0.07)$ were found.

Masson's trichrome and Picrosirius red stains both confirmed the presence of newly formed collagen from day 4 (Fig. 5a). Gradually, more collagen was deposited throughout the experimental period (Figs. 5a-c).

Hyperplasia and early migration of epithelial basal cells were seen from $12 \mathrm{~h}$ (2/8 wounds, $25 \%)$ and complete epithelization was present in wounds being from 18 to 35 days old. This is in agreement with another porcine model based on $2 \times 2 \mathrm{~cm}$ full thickness wounds, in which complete epithelization was achieved on day 28 [18].

Histological features of significant importance for assessing the age of porcine wounds were identified (Table 1, Fig. 6). Age assessment of wounds without granulation tissue should be based on the ratio between neutrophils and macrophages and the presence of angiogenesis and fibroblast hyperplasia (Fig. 6). In wounds containing granulation tissue, the thickness of it was useful for wound age determination and the neutrophil:macrophage ratio, deposition of collagen and 


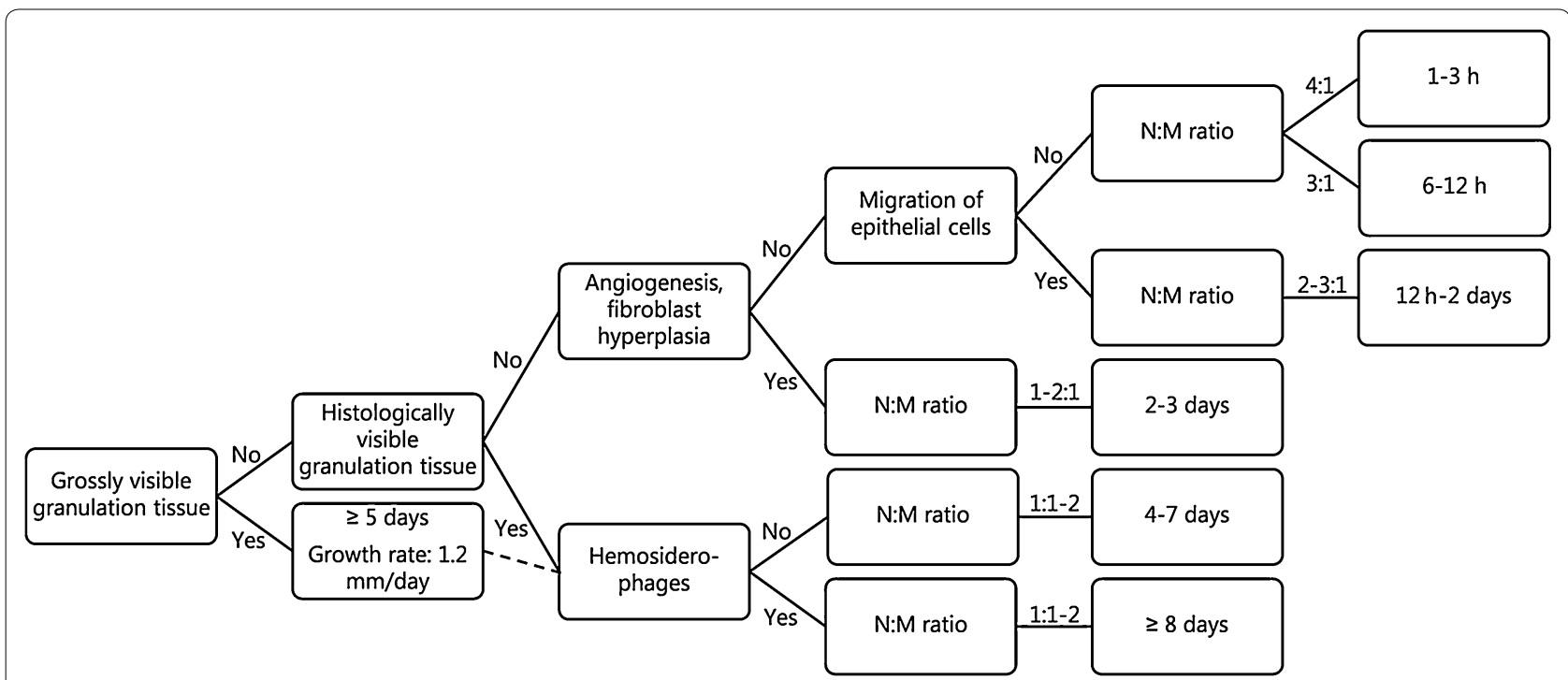

Fig. 6 Decision diagram for determining the age of wounds based on gross and histological evaluation

presence of hemosiderophages can further support the assessment (Fig. 6).

\section{Additional files}

Additional file 1. Sedation and general anesthesia for surgical incision of wounds.

Additional file 2. Anesthesia and reversal of anesthesia for sampling of biopsies.

Additional file 3. Granulation tissue was measured at the center of the wound from the top of the wound bed (not including epidermis) and excluding underlying fat tissue and the fibrous fascia of the muscle.

Additional file 4. Infiltration of neutrophils and macrophages was scored as: (0) absent; (1)<10; (2) 10-20; (3) 21-50; (4) 51-100; (5) 101-200; (6) > 200 using a $40 \times$ objective and 10x ocular with FN $22 \mathrm{~mm}$ in one high power field (HPF) of $0.237 \mathrm{~mm}^{2}$. The scoring was carried out in the HPF with the highest number of neutrophils and macrophages within areas $1-4$ in the wounds (Fig. 2a, b). From each wound, only the highest score was registered, i.e. one neutrophil score and one macrophage score per wound. Location of the wound (1-4), wound age, neutrophil score and macrophage score are presented.

\section{Abbreviations}

SPF: specific pathogen free, i.e. free from infection with Mycoplasma hyopneumoniae, Actinobacillus pleuropneumoniae serotype 1-10 and 12, Porcine Reproductive Respiratory Syndrome, Brachyspira hyodysenteriae, Pasteurella multocida, Sarcoptes Scabiei var. Suis. and Haematopinus suis.

\section{Authors' contributions}

$\mathrm{KB}$ and $\mathrm{HEJ}$ contributed to the design of the study. All authors contributed to the experimental work and the gross and histological evaluation of the tissue. In addition, KB performed the statistical analyses and drafted the manuscript. All authors read and approved the final manuscript.

\section{Acknowledgements}

The authors wish to thank Elisabeth Wairimu Petersen and Betina Gjedsted Andersen for skilled technical assistance with preparation of tissue for histology and immunohistochemistry. Moreover, we wish to thank Frederik Andersen and Dennis Brok for skilled technical assistance with photos and figures.

\section{Competing interests}

The authors declare that they have no competing interests.

\section{Availability of data}

All data generated or analyzed during this study are included in this published article and its additional files.

\section{Consent for publication}

Not applicable.

\section{Ethics approval and consent to participate}

The study and the procedures were approved by the Danish Animal Inspectorate (2013-15-2934-00849) and were carried out in accordance with all institutional, local and national guidelines.

\section{Funding}

The study was funded by University of Copenhagen, Denmark. The funding source had no involvement in the experimental design, analysis and interpretation of the results.

\section{Publisher's Note}

Springer Nature remains neutral with regard to jurisdictional claims in published maps and institutional affiliations.

Received: 16 January 2018 Accepted: 19 May 2018

Published online: 30 May 2018

\section{References}

1. de Siqueira A, Cuevas SEC, Salvagni FA, Maiorka PC. Forensic veterinary pathology. Vet Pathol. 2016;53:979-87.

2. Ressel $\mathrm{L}$, Hetzel U, Ricci E. Blunt force trauma in veterinary forensic pathology. Vet Pathol. 2016;53:941-61.

3. Barington K, Dich-Jørgensen K, Jensen HE. A retrospective study of forensic cases of skin ulcerations in Danish pigs from 2000 to 2014. Acta Vet Scand. 2016:58:48. 
4. Wang JF, Olson ME, Reno CR, Wright JB, Hart DA. The pig as a model for excisional skin wound healing: characterization of the molecular and cellular biology, and bacteriology of the healing process. Comp Med. 2001;51:341-8.

5. Rigal C, Pieraggi MT, Vincent C, Prost C, Bouisou H, Serre G. Healing of full-thickness cutaneous wounds in the pig. I. Immunohistochemical study of epidermo-dermal junction regeneration. J Invest Dermatol. 1991;96:777-85.

6. Wang JF, Olson ME, Reno CR, Kulyk W, Wright JB, Hart DA. Molecular and cell biology of skin wound healing in a pig model. Connect Tissue Res. 2000:41:195-211.

7. Ackermann MR. Inflammation and Healing. In: Zachary JF, McGavin MD, editors. Pathologic basis of veterinary disease. St. Louis: Elsevier Mosby; 2012. p. 89-146.

8. Barington $\mathrm{K}$, Jensen HE. Forensic aspects of incised wounds and bruises in pigs established post-mortem. Res Vet Sci. 2017;112:42-5.

9. Meuten DJ, Moore FM, George JW. Mitotic count and the field of view area. Vet Pathol. 2016;53:7-9.

10. Luna LG. Manual of histological staining methods of the Armed Forces Institute of Pathology. 3rd ed. New York: McGraw-Hill Book Company; 1968.
11. Montes GS, Junqueira LC. The use of the Picrosirius-polarization method for the study of the biopathology of collagen. Mem Inst Oswaldo Cruz. 1991;86:1-11.

12. Jensen HE, Nielsen OL, Agerholm JS, Iburg T, Johansen LK, Johannesson E, et al. A non-traumatic Staphylococcus aureus osteomyelitis model in pigs. In Vivo. 2010;24:257-64.

13. Barington $\mathrm{K}$, Jensen HE. Forensic aspects of agonal wounds in slaughter pigs. In: 35th ESVP Meet. Eur. Soc. Vet. Pathol. 28th ECVP Meet Eur Coll Vet Pathol. 2017.

14. Elgharably H, Roy S, Khanna S, Abas M, DasGhatak P, Das A, et al. A modified collagen gel enhances healing outcome in a preclinical swine model of excisional wounds. Wound Repair Regen. 2013;21:473-81.

15. Betz P. Histological and enzyme histochemical parameters for the age estimation of human skin wounds. Int J Legal Med. 1994;107:60-8.

16. Eming SA, Krieg T, Davidson JM. Inflammation in wound repair: molecular and cellular mechanisms. J Invest Dermatol. 2007;127:514-25.

17. Bouissou H, Pieraggi M, Julian M, Uhart D, Kokolo J. Fibroblasts in dermal tissue repair. Electron microscopic and immunohistochemical study. Int J Dermatol. 1988;27:564-70.

18. Gallant-Behm CL, Hart DA. Genetic analysis of skin wound healing and scarring in a porcine model. Wound Repair Regen. 2006;14:46-54.
Ready to submit your research? Choose BMC and benefit from:

- fast, convenient online submission

- thorough peer review by experienced researchers in your field

- rapid publication on acceptance

- support for research data, including large and complex data types

- gold Open Access which fosters wider collaboration and increased citations

- maximum visibility for your research: over $100 \mathrm{M}$ website views per year

At BMC, research is always in progress.

Learn more biomedcentral.com/submissions 